\begin{tabular}{|c|c|}
\hline \multicolumn{3}{|c|}{ DJS Vol.39 (2018) 9-17 } \\
\hline 1969 & $\begin{array}{c}\text { Delta Journal of Science } \\
\text { Available online at } \\
\text { https://djs.journals.ekb.eg/ }\end{array}$ \\
\hline Research Article & \\
\hline
\end{tabular}

\title{
Probabilistic Mixture Shortage Multi-Source Inventory Model with Varying Holding Cost Under Constraint
}

Hala A. Fergany and Magda A. Gomaa

Mathematics Department, Faculty of Science, Tanta University, Tanta 31527, Egypt.

* Correspondence: Magda A. Gomaa; Mathematics Department, Faculty of Science, Tanta University, Tanta 31527,

Egypt; Email: magda.farag@science.tanta.edu.eg

\section{Key words:}

Probabilistic

inventory model;

Mixture shortage;

Lagrange multiplier

approach;

Varying holding

cost;

Trapezoidal fuzzy

number and

Continuous

distributions.

\section{Abstract:}

This paper proposed a general probabilistic continuous review multiitem, multi-source inventory model with constraint for crisp and fuzzy environment. This constraint on the expected varying holding cost. The demand is a continuous random variable, the distribution of the lead time demand is known and the holding cost is varying. This model is formulated to analyze how the firm can deduce the optimal order quantity and the optimal reorder point for the item and source to reach the main goal of minimizing the expected total cost using a Lagrange multiplier technique. The lead time demand is follow some continuous distributions. Also, an application with real data is analyzed and the goal of minimization the expected total cost is achieved.

\section{Introduction:}

The multi-item, multi-source inventory system is the most general procurement system which may be described as follows; an inventory of i-items is maintained to meet the average demand rates designated , , ,..., . Most of the probabilistic inventory models assume that the units of cost are constant or one of these units is varying. Hundreds of papers and books have been published presenting models for doing this under a wide variety of conditions and assumptions [12] discussed analysis of inventory system [5] explained procurement and inventory system: theory and analysis. An application of the systempoint method to inventory models under continuous review has been studied by [2]. [14] presented inventory model with a mixture of backorders and lost sales. [11] Explained unconstrained probabilistic inventory problems with constant units of cost. [1] Studied the probabilistic multi-item single- source inventory model with varying order cost under two restrictions. [6] Deduced probabilistic single-item inventory problem with varying order cost under two linear constraints. Constrained periodic review probabilistic inventory model with continuous distributions and varying holding cost have been studied by [7]. [13] Discussed the optimal and near -optimal policies for lost sales inventory models with at 
most one replenishment order outstanding. [8] Examined Probabilistic multi-item inventory model with varying mixture shortage cost under restrictions. [3] Deduced a multi-item single-source mixture inventory model involving random lead time and demand with budget constraint and surprise function. [16] Studied a two-demand-class inventory system with lost sales and backorders. [10] Studied Multi-item EOQ model with varying holding cost: a geometric programming approach.

The cost parameters in real inventory systems and other parameters such as price marketing and service elasticity to demand are imprecise and uncertain in nature. So, the notion of fuzziness can be applied to cope with this uncertainly. Since the proposed model is in a fuzzy environment, a fuzzy decision should be made to meet the decision criteria, and the results should be fuzzy. Fuzzy sets introduced by many researchers as a mathematical way of representing impreciseness or vagueness in everyday life. [4] Introduced fuzzy inventory with backorder defuzzification by signed distance method. [9] Deduced Probabilistic periodic review $\langle Q, r\rangle$ inventory model using LaGrange technique and fuzzy adapting particle swarm optimization. [15] Presented deterministic inventory models with a mixture of backorders and lost sales under fuzzy cost. In this paper, we investigate a probabilistic multi-item, multi-source (MIMS) continuous review inventory model with mixture shortage, varying holding unit cost, under the expected varying holding cost constraint for crisp and fuzzy environment. The expected total cost of inventory system is composed of three components (the expected order cost, the expected varying holding cost and the expected mixture shortage cost). The optimal solutions of the order quantity $\left(Q^{*}{ }_{i s}\right)$, the reorder point $\left(r^{*}{ }_{i s}\right)$, which minimize the expected total cost, $\mathrm{E}\left(\mathrm{TC}\left(Q_{i s}, r_{i s}\right)\right)$, using Lagrange technique, are obtained mathematically. Also, the model will be studied when the lead time demand follows Gamma, Weibull, Chi-square, Erlang and Exponential distributions and an application is added to illustrate the model for crisp and fuzzy environment.

\section{Model Notions}

The following notations are adopted for developing our model:

$\bar{D}_{i} \quad$ The average annual demand for the $i^{\text {th }}$ item.

$Q_{i s} \quad$ The decision variable representing the order quantity per cycle for the $i^{\text {th }}$ item and $s^{\text {th }}$ source.

$r_{i s} \quad$ The decision variable representing the reorder point per cycle for the $i^{\text {th }}$ item and $s^{\text {th }}$ source.

$C_{\text {ois }} \quad$ The inventory order cost per unit per cycle for the $i^{\text {th }}$ item and $s^{\text {th }}$ source.

$C_{h i} \quad$ The inventory holding cost per unit per cycle for the $i^{t h}$ item.

$C_{h i}\left(Q_{i s}\right)$ The varying holding cost per unit per cycle for the $i^{\text {th }}$ item and $s^{\text {th }}$ source.

$C_{b i} \quad$ The inventory backorder cost per unit per cycle for the $i^{t h}$ item.

$C_{l i} \quad$ The inventory lost sales cost per unit per cycle for the $i^{\text {th }}$ item.

$k_{h i} \quad$ The limitation on the expected annual holding cost for the $i^{\text {th }}$ item.

$\lambda_{\text {his }} \quad$ Lagrange multiplier for the $i^{t h}$ item and $s^{\text {th }}$ source.

$E_{i s}(O C)$ The expected order cost for the $i^{\text {th }}$ item and $s^{t h}$ source.

$E_{i s}(H C)$ The expected varying holding cost for the $i^{\text {th }}$ item and $s^{\text {th }}$ source.

$E_{i s}(B C)$ The expected backorder cost for the $i^{\text {th }}$ item and $s^{\text {th }}$ source.

$E_{i s}(L C)$ The expected lost sales cost for the $i^{\text {th }}$ item and $s^{\text {th }}$ source.

$E_{i s}(S C)$ The expected shortage cost $=$ $E_{i s}(\mathrm{BC})+E_{i s}(\mathrm{LC})$ for the $i^{\text {th }}$ item and $s^{\text {th }}$ source.

$\operatorname{Min} E(T C)$ The minimum expected annual total cost for the $i^{\text {th }}$ item and $s^{t h}$ source

$$
=\sum_{\mathrm{i}=1}^{\mathrm{m}} \min _{\mathrm{s}} \mathrm{ETC}_{\mathrm{is}}, \quad \mathrm{s}=1,2, \ldots, \mathrm{v}
$$




\section{Model Development}

The following assumptions are made for developing the mathematical model:

(1) The expected order cost is given by:

$$
E_{i s}(O C)=C_{\text {ois }} \frac{\bar{D}_{i}}{Q_{i s}}
$$

(2) The varying expected holding cost is given by:

$$
\begin{aligned}
& E_{i s}(H C)=C_{h i}\left(Q_{i s}\right) n_{i} \bar{H}_{i} \\
& =C_{h i}\left(\alpha+\beta Q_{i s}{ }^{-1}\right)\left[\frac{Q_{i s}}{2}+r_{i s}-\mu+\left(1-\gamma_{i}\right) \bar{S}\left(r_{i s}\right)\right]
\end{aligned}
$$

Where, the varying holding cost per unit per cycle is a function of the order quantity which takes the following form:

$C_{h i}\left(Q_{i s}\right)=C_{h i}\left(\alpha+\beta Q_{i s}{ }^{-1}\right), \alpha, \beta>0$

Also, $H_{i}$ represents the average inventory level during the cycle. $\bar{H}_{i}=\frac{H_{i}}{n_{i}}, n_{i}$ is average of cycle per year and $n_{i}=\frac{\bar{D}_{i}}{Q_{i s}}$

(3) The expected shortage cost is the mixture of the expected backorder cost and the expected lost sales cost as follows:

$E_{i s}(S C)=E_{i s}(B C)+E_{i s}(L C)$

Where,

$E_{i s}(B C)=C_{b i} \gamma_{i} \frac{\bar{D}_{i}}{Q_{i s}} \bar{S}\left(r_{i s}\right)$ and

$\mathrm{E}_{i s}(L C)=C_{l i}\left(1-\gamma_{i}\right) \frac{\bar{D}_{i}}{Q_{i s}} \bar{S}\left(r_{i s}\right), 0 \leq \gamma_{\mathrm{i}} \leq 1$

The objective is to minimize the relevant expected total cost function (i.e. the sum of the expected order cost, the expected varying holding cost, the expected backorder cost and the expected lost sales cost) which, according to the previous assumptions of the model is

$$
\begin{aligned}
& \mathrm{E}\left(\mathrm{TC}\left(\mathrm{Q}_{\mathrm{is}}, \mathrm{r}_{\mathrm{is}}\right)\right)=\mathrm{E}\left(\mathrm{TC}_{\mathrm{is}}\right) \\
& =\sum_{\mathrm{i}=1}^{\mathrm{m}}\left[\mathrm{E}_{\mathrm{is}}(O C)+E_{i s}(H C)+E_{i s}(S C)\right] \\
& \min \mathrm{E}(\mathrm{TC})=\min \sum E\left(T C_{i s}\right)
\end{aligned}
$$

$$
=\min \sum_{i=1}^{m}\left[\begin{array}{c}
\frac{C_{o i s} \bar{D}_{i}}{Q_{i s}}+C_{h i}\left(\alpha+\beta Q_{i s}{ }^{-1}\right)\left[\frac{Q_{i s}}{2}+r_{i s}\right. \\
\left.-\mu+\left(1-\gamma_{i}\right) \bar{S}\left(r_{i s}\right)\right]+C_{b i} \frac{\gamma_{i} \bar{D}_{i}}{Q_{i s}} \bar{S}\left(r_{i s}\right) \\
+C_{l i}\left(1-\gamma_{\mathrm{i}}\right) \frac{\bar{D}_{i}}{Q_{i s}} \bar{S}\left(r_{i s}\right)
\end{array}\right]
$$

Subject to the following expected varying holding cost constraint:

$$
\sum_{i=1}^{m}\left[E_{i s}(H C) \leq k_{h i}\right]
$$

Then to solve this function, under the above constraint, the Lagrange multipliers technique should be used as follows:

$$
\begin{array}{r}
L=\sum_{i=1}^{m}\left[\mathrm{E}\left(T C_{i s}\right)+\lambda_{\text {his }}\left\{E_{i s}(H C)-k_{h i}\right\}\right], \lambda_{\text {his }}>0 \\
=\sum_{i=1}^{m}\left[\begin{array}{c}
\frac{C_{\text {ois }} \bar{D}_{i}}{Q_{i s}}+C_{h i}\left(\alpha+\beta Q_{i s}{ }^{-1}\right)\left[\frac{Q_{i s}}{2}+r_{i s}-\mu\right. \\
\left.+\left(1-\gamma_{i}\right) \bar{S}\left(r_{i s}\right)\right]+C_{b i} \frac{\gamma_{i} \bar{D}_{i}}{Q_{i s}} \bar{S}\left(r_{i s}\right) \\
+C_{l i}\left(1-\gamma_{\mathrm{i}}\right) \frac{\bar{D}_{i}}{Q_{i s}} \bar{S}\left(r_{i s}\right) \\
+\lambda_{h i s}\left[C _ { h i } ( \alpha + \beta Q _ { i s } { } ^ { - 1 } ) \left\{\frac{Q_{i s}}{2}+r_{i s}\right.\right. \\
\left.\left.-\mu+\left(1-\gamma_{i}\right) \bar{S}\left(r_{i s}\right)\right\}-K_{\mathrm{hi}}\right]
\end{array}\right]
\end{array}
$$

The optimal values of the order quantity $\left(Q_{i s}{ }^{*}\right)$ and reorder point $\left(r_{i s}{ }^{*}\right)$ which are minimizing the expected total costfor the $i^{\text {th }}$ item and $s^{\text {th }}$ source, can be calculated by setting each of the corresponding first partial derivatives of (3) with respect to the two decision variables equal to zero, then the following is obtained:

$$
\begin{aligned}
\frac{\partial \mathrm{L}}{\partial \mathrm{Q}_{\mathrm{is}}}= & -\frac{\mathrm{C}_{\mathrm{ois}} \overline{\mathrm{D}}_{\mathrm{i}}}{\mathrm{Q}_{\mathrm{is}}{ }^{2}}-\frac{\mathrm{C}_{\mathrm{hi}} \beta}{\mathrm{Q}_{\mathrm{is}}{ }^{2}}\left[\begin{array}{c}
\frac{\mathrm{Q}_{\mathrm{is}}}{2}+\mathrm{r}_{\mathrm{is}}-\mu \\
+\left(1-\gamma_{i}\right) \bar{S}\left(r_{i s}\right)
\end{array}\right] \\
& +\frac{\mathrm{C}_{\mathrm{hi}}\left(\alpha+\beta \mathrm{Q}_{\mathrm{is}}{ }^{-1}\right)}{2}-\frac{C_{b i} \gamma_{i} \bar{D}_{i}}{Q_{i s}{ }^{2}} \bar{S}\left(r_{i s}\right) \\
& -\frac{\mathrm{C}_{\mathrm{lii}}\left(1-\gamma_{\mathrm{i}}\right) \overline{\mathrm{D}}_{\mathrm{i}}}{\mathrm{Q}_{\mathrm{is}}{ }^{2}} \overline{\mathrm{S}}\left(\mathrm{r}_{\mathrm{is}}\right)-\lambda_{\mathrm{his}}\left[\mathrm{C}_{\mathrm{hi}} \frac{\beta}{\mathrm{Q}_{\mathrm{is}}{ }^{2}}\left[\begin{array}{c}
\frac{\mathrm{Q}_{\mathrm{is}}}{2}+\mathrm{r}_{\mathrm{is}}-\mu \\
+\left(1-\gamma_{\mathrm{i}}\right) \overline{\mathrm{S}}\left(\mathrm{r}_{\mathrm{is}}\right)
\end{array}\right]\right. \\
& +\frac{\lambda_{h i s} C_{h i}\left(\alpha+\beta Q_{i s}{ }^{-1}\right)}{2},
\end{aligned}
$$


Then, we can obtain the optimal order quantity and the optimal reorder point from the following equations:

$\alpha \mathrm{AQ}_{\mathrm{is}}{ }^{* 2}=\mathrm{B}+2 \mathrm{~A} \beta\left\{\mathrm{r}_{\mathrm{is}}{ }^{*}-\mu+\left(1-\gamma_{\mathrm{i}}\right) \overline{\mathrm{S}}\left(\mathrm{r}_{\mathrm{is}}{ }^{*}\right)\right\}$ $+2 \mathrm{MS}\left(\mathrm{r}_{\mathrm{is}}{ }^{*}\right)$

Where $\mathrm{A}=\left(1+\lambda_{\text {his }}\right) \mathrm{C}_{\mathrm{hi}}, \mathrm{B}=2 \mathrm{C}_{\text {ois }} \overline{\mathrm{D}}_{\mathrm{i}}$,

$\mathrm{M}=\left[C_{b i} \gamma_{i} \bar{D}_{i}+C_{l i}\left(1-\gamma_{i}\right) \bar{D}_{i}\right]$

i.e.

$\alpha A Q_{i s}{ }^{* 2}-B-2 \mathrm{~A} \beta\left\{r_{i s}{ }^{*}-\mu\right.$

$\left.+\left(1-\gamma_{i}\right) \bar{S}\left(r_{i s}^{*}\right)\right\}-2 \mathrm{M} \bar{S}\left(r_{i s}^{*}\right)=0$

also,

$\frac{\partial \mathrm{L}}{\partial \mathrm{r}_{\mathrm{is}}}=\mathrm{c}_{\mathrm{hi}}\left(\alpha+\beta \mathrm{Q}_{\mathrm{is}}{ }^{-1}\right)\left[1-\left(1-\gamma_{\mathrm{i}}\right) \mathrm{R}\left(\mathrm{r}_{\mathrm{is}}\right)-\right.$

$\frac{\mathrm{c}_{\mathrm{bi}} \gamma_{\mathrm{i}} \overline{\mathrm{D}}_{\mathrm{i}}}{\mathrm{Q}_{\mathrm{is}}} \mathrm{R}\left(\mathrm{r}_{\mathrm{is}}\right)-\frac{\mathrm{C}_{\mathrm{li}}\left(1-\gamma_{\mathrm{i}}\right) \overline{\mathrm{D}}_{\mathrm{i}}}{\mathrm{Q}_{\mathrm{is}}} \mathrm{R}\left(\mathrm{r}_{\mathrm{is}}\right)+\lambda_{\text {his }}\left[\mathrm{C}_{\mathrm{hi}}(\alpha\right.$ $\left.+\beta \mathrm{Q}_{\mathrm{is}}{ }^{-1}\right)\left\{\left(1-\left(1-\gamma_{\mathrm{i}}\right) \mathrm{R}\left(\mathrm{r}_{\mathrm{is}}\right)\right\}\right]$

$\mathrm{R}\left(\mathrm{r}_{\mathrm{is}}{ }^{*}\right)=\frac{\mathrm{A}\left(\alpha \mathrm{Q}_{\mathrm{is}}{ }^{*}+\beta\right)}{\mathrm{M}+\mathrm{A}\left(1-\gamma_{\mathrm{i}}\right)\left(\alpha \mathrm{Q}_{\mathrm{is}}{ }^{*}+\beta\right)}$

and we can prove that:

$\left\{\begin{array}{c}{\left[\frac{\partial^{2} L}{\partial Q_{i}{ }^{2}}\right]\left[\frac{\partial^{2} L}{\partial r_{i}{ }^{2}}\right]-\left[\frac{\partial^{2} L}{\partial Q_{i} \partial r_{i}}\right]^{2}>0} \\ \frac{\partial^{2} L}{\partial Q_{i}{ }^{2}} \text { or, } \frac{\partial^{2} L}{\partial r_{i}{ }^{2}}>0\end{array}\right.$

It is clearly that there is no closed form solution, then an iterative method must be used to determine $Q_{i s}{ }^{*}$ and $r_{i s}{ }^{*}$ (as the following algorithm) which are used to determine the minimum expected total cost.

\section{Algorithm}

Step 1: Input all the inventory model data for example, expected demand value, order unit cost, holding unit cost, mean, etc. at one $\beta$ value and assumption value of $\lambda$ and put, $r_{0}=\mu$ as an initial value so, $S_{0}=0$, then calculate the first order quantity $Q_{1}$.

Step 2: Use the calculated order quantity in step 1 to calculate $r_{1}$ and $S_{1}$.

Step 3: Use the calculated $r_{1}$ and $S_{1}$ in step 2 to calculate a new order quantity $Q_{2}$.
Step 4: Repeat steps 1 and 2. If two values of respectively calculated order quantity are equaled, then it is the optimal $Q^{*}$ and $r^{*}$.

Step 5: Using the calculated optimal order quantity $Q^{*}$ and optimal reorder point $r^{*}$ to calculate the expected total cost.

Step 6: Repeat all steps at changes values of $\lambda$ to be the condition is active. If the condition is active, then it is the minimum expected total cost at this value of $\beta$.

Step 7: Repeat all steps at other values of $\beta$.

\section{The Model When all Parameters are Trapezoidal Fuzzy Numbers}

Consider inventory cost coefficients in the model are fuzzy in nature. Therefore, the decision variables and the objective function should be fuzzy as well, and we are interested in driving the membership functions of $\mathrm{E}(\mathrm{TC})$ by solving the model via Lagrange multipliers technique according to the assumptions, we can investigate the following formulation for the fuzzy inventory model:

$$
=\sum_{\mathrm{i}=1}^{\mathrm{E}\left(\widetilde{\mathrm{TC}}_{\mathrm{is}}\right)}\left[\begin{array}{c}
\frac{\tilde{\mathrm{C}}_{\text {ois }} \overline{\mathrm{D}}_{\mathrm{i}}}{\mathrm{Q}_{\mathrm{is}}}+\tilde{\mathrm{C}}_{\mathrm{hi}}\left(\alpha+\beta \mathrm{Q}_{\mathrm{is}}{ }^{-1}\right)\left[\frac{\mathrm{Q}_{\text {is }}}{2}+\mathrm{r}_{\mathrm{is}}-\mu\right. \\
\left.+\left(1-\gamma_{\mathrm{i}}\right) \overline{\mathrm{S}}\left(\mathrm{r}_{\mathrm{is}}\right)\right] \\
+\tilde{\mathrm{C}}_{\mathrm{bi}} \frac{\gamma_{\mathrm{i}} \overline{\mathrm{D}}_{\mathrm{i}}}{\mathrm{Q}_{\mathrm{is}}} \overline{\mathrm{S}}\left(\mathrm{r}_{\mathrm{is}}\right) \\
+\tilde{\mathrm{C}}_{\mathrm{li}}\left(1-\gamma_{\mathrm{i}}\right) \frac{\overline{\mathrm{D}}_{\mathrm{i}}}{\mathrm{Q}_{\mathrm{is}}} \overline{\mathrm{S}}\left(\mathrm{r}_{\mathrm{is}}\right)
\end{array}\right]
$$

Subject to the following varying holding cost constraint:

$\sum_{\mathrm{i}=1}^{\mathrm{m}} \tilde{\mathrm{C}}_{\mathrm{hi}}\left(\alpha+\beta \mathrm{Q}_{\mathrm{is}}{ }^{-1}\right)\left[\frac{\mathrm{Q}_{\mathrm{is}}}{2}+\mathrm{r}_{\mathrm{is}} \leq \sum_{\mathrm{i}=1}^{\mathrm{m}} \mathrm{k}_{\mathrm{hi}}\right.$
$\left.-\mu+\left(1-\gamma_{\mathrm{i}}\right) \overline{\mathrm{S}}\left(\mathrm{r}_{\mathrm{is}}\right)\right]$

To solve this inventory model using Lagrange multipliers technique, we should find the left and the right shape functions of the objective function and decision variables, by find the upper bound and the lower bound of the objective function, i.e. $\tilde{L}_{v}(\alpha)$ and $\tilde{L}_{u}(\alpha)$ respectively. Recall that $\tilde{L}_{v}(\alpha)$ and $\tilde{L}_{u}(\alpha)$ represent the smallest and largest values (The 
left and right $\alpha$ cuts) of the optimal objective function $\tilde{L}(\alpha)$.

Consider the model when all parameters are trapezoidal fuzzy numbers as given below

$\tilde{C}_{\text {ois }}=\left(C_{\text {ois }}-\delta_{1 i}, C_{\text {ois }}-\delta_{2 i}, C_{\text {ois }}+\delta_{3 i}, C_{\text {ois }}+\delta_{4 i}\right)$,

$\tilde{C}_{h i}=\left(C_{h i}-\delta_{5 i}, C_{h i}-\delta_{6 i}, C_{h i}+\delta_{7 i}, C_{h i}+\delta_{8 i}\right)$,

$\tilde{C}_{b i}=\left(C_{b i}-\delta_{9 i}, C_{b i}-\delta_{10 i}, C_{b i}+\delta_{11 i}, C_{b i}+\delta_{12 i}\right)$,

and $\tilde{C}_{l i}=\left(C_{l i}-\delta_{13 i}, C_{l i}-\delta_{14 i}, C_{l i}+\delta_{15 i}, C_{l r}+\delta_{16 i}\right)$.

Where $\delta_{i}, \mathrm{i}=1,2, \ldots, 16$ are arbitrary positive numbers satisfy the following restrictions:

$\tilde{C}_{\text {ois }}>\delta_{1 i}>\delta_{2 i}, \delta_{3 i}<\delta_{4 i}$,

$\tilde{C}_{h i}>\delta_{5 i}>\delta_{6 i}, \delta_{7 i}<\delta_{8 i}$,

$\tilde{C}_{b i}>\delta_{9 i}>\delta_{10 i}, \delta_{11 i}<\delta_{12 i}$ and

$\tilde{C}_{l i}>\delta_{13 i}>\delta_{14 i}, \delta_{15 i}<\delta_{16 i}$.

The left and right limits $\alpha$ cuts of

$C_{o i s}, C_{h i}, C_{b i}, C_{l i}$ are given by:

$\tilde{C}_{o i s_{v}}(\alpha)=C_{o i s}-\delta_{1 i}+\left(\delta_{1 i}-\delta_{2 i}\right) \alpha, \tilde{C}_{o i s_{u}}(\alpha)=$

$C_{\text {ois }}+\delta_{4 i}-\left(\delta_{4 i}-\delta_{3 i}\right) \alpha$,

$\tilde{C}_{h i_{v}}(\alpha)=C_{h i}-\delta_{5 i}+\left(\delta_{5 i}-\delta_{6 i}\right) \alpha, \tilde{C}_{h i_{u}}(\alpha)=$

$C_{h i}+\delta_{8 i}-\left(\delta_{8 i}-\delta_{7 i}\right) \alpha$,

$\tilde{C}_{b i_{v}}(\alpha)=C_{b i}-\delta_{9 i}+\left(\delta_{9 i}-\delta_{10 i}\right) \alpha, \tilde{C}_{b i_{u}}(\alpha)=C_{b i}+$

$\delta_{12 i}-\left(\delta_{12 i}-\delta_{11 i}\right) \alpha$ and

$\tilde{C}_{l i_{v}}(\alpha)=C_{l i}-\delta_{13 i}+\left(\delta_{13 i}-\delta_{14 i}\right) \alpha, \tilde{C}_{l l_{u}}(\alpha)=$

$C_{l i}+\delta_{16 i}-\left(\delta_{16 i}-\delta_{15 i}\right) \alpha$.

Where $\tilde{C}_{\text {ois }}=\frac{1}{4}\left[4 C_{\text {ois }}-\delta_{1 i}-\delta_{2 i}+\delta_{3 i}+\delta_{4 i}\right], \tilde{C}_{h i}$ $=\frac{1}{4}\left[4 C_{h i}-\delta_{5 i}-\delta_{6 i}+\delta_{7 i}+\delta_{8 i}\right], \tilde{C}_{b i}=\frac{1}{4}\left[4 C_{b i}-\delta_{9 i}-\right.$ $\left.\delta_{10 i}+\delta_{11 i}+\delta_{12 i}\right]$ and $\tilde{C}_{l i}=\frac{1}{4}\left[4 C_{l i}-\delta_{13 i}-\delta_{14 i}+\right.$ $\left.\delta_{15 i}+\delta_{16 i}\right]$.

Using approximated value of trapezoidal fuzzy numbers which observe in Figure 1.

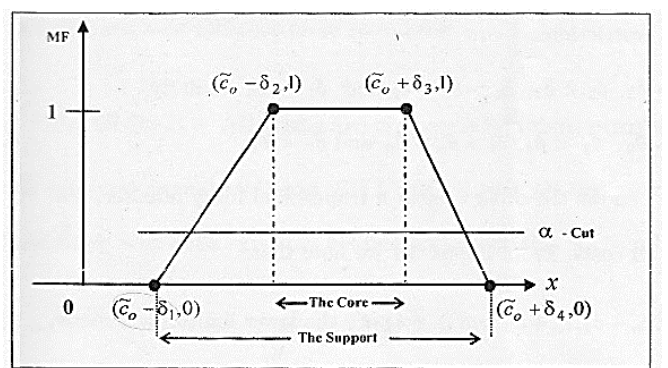

Figure (1): Order cost as trapezoidal fuzzy number.
By using the Lagrange multiplier technique, the above fuzzy system of equations (6) and (7) reduces to

$$
\tilde{L}=\sum_{i=1}^{m}\left[\begin{array}{c}
\frac{\tilde{\mathrm{C}}_{\mathrm{ois}} \bar{D}_{i}}{Q_{i s}}+\tilde{\mathrm{C}}_{\mathrm{hi}}\left(\alpha+\beta Q_{i s}{ }^{-1}\right)\left[\frac{Q_{i s}}{2}+r_{i s}\right. \\
\left.-\mu+\left(1-\gamma_{i}\right) \bar{S}\left(r_{i s}\right)\right] \\
+\tilde{\mathrm{C}}_{\mathrm{bi}} \frac{\gamma_{i} \bar{D}_{i}}{Q_{i s}} \bar{S}\left(r_{i s}\right) \\
+\tilde{\mathrm{C}}_{\mathrm{li}}\left(1-\gamma_{\mathrm{i}}\right) \frac{\bar{D}_{i}}{Q_{i s}} \bar{S}\left(r_{i s}\right) \\
+\lambda_{h i s}\left[\tilde { \mathrm { C } } _ { \mathrm { hi } } ( \alpha + \beta Q _ { i s } { } ^ { - 1 } ) \left\{\frac{Q_{i s}}{2}+r_{i s}\right.\right. \\
\left.\left.-\mu+\left(1-\gamma_{i}\right) \bar{S}\left(r_{i s}\right)\right\}-K_{\mathrm{hi}}\right]
\end{array}\right]
$$

Then, we can obtain the optimal order quantity and the optimal reorder point from the following equations:

$$
\begin{aligned}
& \alpha A 1 Q_{i s}{ }^{* 2}-B 1-2 \mathrm{~A} 1 \beta\left\{r_{i s}{ }^{*}-\mu\right. \\
& \left.\quad+\left(1-\gamma_{i}\right) \bar{S}\left(r_{i s}{ }^{*}\right)\right\}-2 \mathrm{M} 1 \bar{S}\left(r_{i s}{ }^{*}\right)=0
\end{aligned}
$$

Where $\mathrm{A} 1=\left(1+\lambda_{\text {his }}\right) \tilde{C}_{h i}, \mathrm{~B} 1=2 \tilde{C}_{\text {ois }} \bar{D}_{i}, \mathrm{M} 1=$ $\left[\tilde{C}_{b i} \gamma_{i} \bar{D}_{i}+\tilde{C}_{l i}\left(1-\gamma_{i}\right) \bar{D}_{i}\right]$

and

$\mathrm{R}\left(r_{i s}{ }^{*}\right)=\frac{\mathrm{A} 1\left(\alpha \mathrm{Q}_{\mathrm{is}}{ }^{*}+\beta\right)}{\mathrm{M} 1+\mathrm{A} 1\left(1-\gamma_{\mathrm{i}}\right)\left(\alpha \mathrm{Q}_{\mathrm{is}}{ }^{*}+\beta\right)}$

By using the algorithm, we can deduce the optimal values $Q_{i s}{ }^{*}, r_{i s}{ }^{*}$

Then, the model will be studied for crisp and fuzzy environment when the lead time demand follows some continuous distributions such as Gamma, Weibull, Chi-square, Erlang and Exponential distributions.

\section{The Model with Continuous Distributions}

Assume that the lead time demand follows some continuous distributions as follows:

\subsection{The Gamma Distribution:}

If the lead time demand follows the Gamma distribution with parameters $p, \theta$, the density function is given by:

$\mathrm{f}(\mathrm{x})=\frac{\theta e^{-\theta x}(\theta x)^{p-1}}{\Gamma(p)}, 0<\mathrm{x}<\infty$ 
and the reliability function is given by:

$\mathrm{R}(\mathrm{r})=\int_{r}^{\infty} f(x) d x=\frac{\Gamma(p, \theta r)}{\Gamma(p)}$, where

$\Gamma(\mathrm{p}, \theta \mathrm{r})$ : upper incomplete Gamma

$R(r)=e^{-\theta r} \sum_{j=0}^{p-1} \frac{(\theta r)^{j}}{j !}$

and the expected shortage quantity is given by:

$\bar{S}(\mathrm{r})=\int_{\mathrm{r}}^{\infty}(\mathrm{x}-\mathrm{r}) \mathrm{f}(\mathrm{x}) \mathrm{dx}=\mathrm{e}^{-\theta \mathrm{r}}\left[\frac{\mathrm{p}}{\theta} \frac{(\theta \mathrm{r})^{\mathrm{p}}}{\mathrm{p} !}-\mathrm{r}\right] \sum_{\mathrm{j}=0}^{\mathrm{p}-1} \frac{(\theta \mathrm{r})^{\mathrm{j}}}{\mathrm{j} !}$

So, the expected total cost can be minimized mathematically by substituting from (13) and (12) in to (4) and (5), respectively, for any $i^{\text {th }}$ item and $s^{\text {th }}$ source, it is found that the optimal values $Q_{i s}{ }^{*}$ and $r_{i s}{ }^{*}$ are given by:

$\alpha \mathrm{AQ}_{\mathrm{is}}{ }^{* 2}-\mathrm{B}-2 \mathrm{~A} \beta\left\{\mathrm{r}_{\mathrm{is}}{ }^{*}-\mu+\left(1-\gamma_{\mathrm{i}}\right)\left[\mathrm{e}^{-\theta \mathrm{r}}\left(\frac{\mathrm{p}}{\theta} \frac{(\theta \mathrm{r})^{\mathrm{p}}}{\mathrm{p} !}-\right.\right.\right.$

r) $\left.\left.\sum_{\mathrm{j}=0}^{\mathrm{p}-1} \frac{(\theta \mathrm{r})^{\mathrm{j}}}{\mathrm{j} !}\right]\right\}-2 \mathrm{M}\left[\mathrm{e}^{-\theta \mathrm{r}}\left(\frac{\mathrm{p}}{\theta} \frac{(\theta \mathrm{r})^{\mathrm{p}}}{\mathrm{p} !}-\mathrm{r}\right) \sum_{\mathrm{j}=0}^{\mathrm{p}-1} \frac{(\theta \mathrm{r})^{\mathrm{j}}}{\mathrm{j} !}\right]=0$

$\mathrm{R}\left(\mathrm{r}_{\mathrm{is}}{ }^{*}\right)=\frac{\mathrm{A}\left(\alpha \mathrm{Q}_{\mathrm{is}}{ }^{*}+\beta\right)}{\mathrm{M}+\mathrm{A}\left(1-\gamma_{\mathrm{i}}\right)\left(\alpha \mathrm{Q}_{\mathrm{is}}{ }^{*}+\beta\right)}=\mathrm{e}^{-\theta \mathrm{r}} \sum_{\mathrm{j}=0}^{\mathrm{p}-1} \frac{(\theta \mathrm{r})^{\mathrm{j}}}{\mathrm{j} !}$

Hence,

If we put $\mathrm{p}=\frac{n}{2}, \theta=\frac{1}{2}$ in equation (11), we found the lead time demand follows the Chisquare distribution, then equations (12) and (13) will be has the following form:

$$
\begin{aligned}
& R(\mathrm{r})=\int_{\mathrm{r}}^{\infty} \mathrm{f}(\mathrm{x}) \mathrm{dx}=\mathrm{e}^{-\mathrm{r} / 2} \sum_{\mathrm{j}=0}^{\frac{\mathrm{n}}{2^{-}}-\frac{(\mathrm{r} / 2)^{\mathrm{j}}}{\mathrm{j} !}} \\
& \bar{S}(r)=\int_{r}^{\infty}(x-r) f(x) d x= \\
& e^{-r / 2}\left[n \frac{(r / 2)^{\frac{n}{2}}}{(n / 2) !}-\mathrm{r}\right] \sum_{\mathrm{j}=0}^{\frac{\mathrm{n}}{2}-1} \frac{(\mathrm{r} / 2)^{\mathrm{j}}}{\mathrm{j} !}
\end{aligned}
$$

The optimal values of the order quantity $\left(Q_{i s}{ }^{*}\right)$ and reorder point $\left(r_{i s}{ }^{*}\right)$ which are minimizing the expected total cost for the $i^{\text {th }}$ item and $s^{t h}$ source, can be calculated by substituting from (17) and (16) in to (4) and (5), respectively, it is found that the optimal values $Q_{i s}{ }^{*}$ and $r_{i s}{ }^{*}$ are given by:

$$
\begin{aligned}
& \alpha A Q_{i s}{ }^{* 2}-B-2 A \beta\left[r_{i s}{ }^{*}-\mu+(1-\right. \\
& \gamma_{i}\left\{e^{-r / 2}\left(n \frac{(r / 2)^{\frac{n}{2}}}{(n / 2) !}-r\right) \sum_{j=0}^{\frac{n}{2}-1} \frac{(r / 2)^{j}}{j !}\right\}- \\
& 2 M\left[e^{-r / 2}\left(n \frac{(r / 2)^{\frac{n}{2}}}{(n / 2) !}-r\right) \sum_{j=0}^{\frac{n}{2}-1} \frac{(r / 2)^{j}}{j !}\right]=0
\end{aligned}
$$

$R\left(r_{i s}{ }^{*}\right)=\frac{A\left(\alpha Q_{i s}{ }^{*}+\beta\right)}{M+A\left(1-\gamma_{i}\right)\left(\alpha Q_{i s}{ }^{*}+\beta\right)}=e^{-r} / 2 \sum_{j=0}^{\frac{n}{2}-1} \frac{(r / 2)^{j}}{j !}$

which represent the optimal values when the lead time demand follows the Chi-square distribution with parameter $\mathrm{n}$.

Also,

If we put $\mathrm{p}=\mathrm{q}$, where $\mathrm{q}$ is a positive integer number, in equation (11), we found the lead time demand follows the Erlang distribution and it is found that the optimal values $Q_{i s}{ }^{*}$ and $r_{i s}{ }^{*}$ are given by:

$$
\begin{aligned}
& \alpha A Q_{i s}{ }^{* 2}-\mathrm{B}-2 \mathrm{~A} \beta\left\{r_{i s}{ }^{*}-\mu+(1-\right. \\
& \left.\left.\gamma_{i}\right)\left[e^{-\theta r}\left(\frac{q}{\theta} \frac{(\theta r)^{q}}{q !}-\mathrm{r}\right) \sum_{j=0}^{q-1} \frac{(\theta r)^{j}}{j !}\right]\right\}- \\
& 2 M\left[e^{-\theta r}\left(\frac{q}{\theta} \frac{(\theta r)^{q}}{q !}-\mathrm{r}\right) \sum_{j=0}^{q-1} \frac{(\theta r)^{j}}{j !}\right]=0 \\
& \mathrm{R}\left(\mathrm{r}_{\mathrm{is}}{ }^{*}\right)=\frac{\mathrm{A}\left(\alpha \mathrm{Q}_{\mathrm{is}}{ }^{*}+\beta\right)}{\mathrm{M}+\mathrm{A}\left(1-\gamma_{\mathrm{i}}\right)\left(\alpha \mathrm{Q}_{\mathrm{is}}{ }^{*}+\beta\right)}=e^{-\theta r} \sum_{j=0}^{q-1} \frac{(\theta r)^{j}}{j !}
\end{aligned}
$$

which represent the optimal values when $\mathrm{X}$ follows the Erlang distribution with parameter $\theta$, q.

And,

If we putp $=1$, in equation (11), we found the lead time demand follows the Exponential distribution and it is found that the optimal values $Q_{i s}{ }^{*}$ and $r_{i s}{ }^{*}$ are given by:

$\alpha A Q_{i s}{ }^{* 2}-\mathrm{B}-2 \mathrm{~A} \beta\left\{r_{i s}{ }^{*}-\mu+\left(1-\gamma_{i}\right)\left[\frac{1}{\theta} e^{-\theta r}\right]\right\}-$

$2 \mathrm{M}\left[\frac{1}{\theta} e^{-\theta r}\right]=0$,

$\mathrm{R}\left(r_{i s}{ }^{*}\right)=\frac{A\left(\alpha Q_{i s}{ }^{*}+\beta\right)}{M+A\left(1-\gamma_{i}\right)\left(\alpha Q_{i s}{ }^{*}+\beta\right)}=e^{-\theta r}$

Which represent the optimal values when $\mathrm{X}$ follows the Exponential distribution with parameter $\theta$.

\subsection{The Weibull Distribution:}

If the lead time demand follows the Weibull distribution with parameters $\sigma, \eta$, the density function is given by:

$$
\mathrm{f}(\mathrm{x})=\frac{\eta}{\sigma}\left(\frac{x}{\sigma}\right)^{\mathrm{n}-1} e^{-\left(\frac{x}{\sigma}\right)^{\eta}}, \quad \mathrm{x}>0
$$


and the reliability function is given by:

$\mathrm{R}(\mathrm{r})=\int_{r}^{\infty} f(x) d x=e^{-\left(\frac{r}{\sigma}\right)^{n}}$

and the expected shortage quantity is given by:

$\bar{S}(r)=\int_{r}^{\infty}(x-r) f(x) d x$
$=\sigma\left(\frac{1}{\eta}\right) ! e^{-\left(\frac{r}{\sigma}\right)^{\eta}} \sum_{j=0}^{1 / \eta} \frac{\left[(r / \sigma)^{\eta}\right]^{j}}{j !}-r e^{-\left(\frac{r}{\sigma}\right)^{\eta}}$

The optimal values of the order quantity $\left(Q_{i s}{ }^{*}\right)$ and reorder point $\left(r_{i s}{ }^{*}\right)$ which are minimizing the expected total costfor the $i^{\text {th }}$ item and $s^{\text {th }}$ source, can be calculated by substituting from (24) and (23) in to (4) and (5), respectively, it is found that the optimal values $Q_{i s}{ }^{*}$ and $r_{i s}{ }^{*}$ are given by:

$\alpha A Q_{i s}{ }^{* 2}-B-2 A \beta\left[r_{i s}{ }^{*}-\mu+\right.$

$\left(1-\gamma_{i}\right)\left\{\sigma\left(\frac{1}{\eta}\right) ! e^{-\left(\frac{r}{\sigma}\right)^{\eta}} \sum_{j=0}^{1 / \eta\left[(r / \sigma)^{\eta}\right]} \frac{j}{j !}-\right.$

$\left.\left.\left.r e^{-\left(\frac{r}{\sigma}\right)^{\eta}}\right\} r e^{-\left(\frac{r}{\sigma}\right)^{\eta}}\right\}\right]-2 \mathrm{M}\left[\sigma\left(\frac{1}{\eta}\right) ! e^{-\left(\frac{r}{\sigma}\right)^{\eta}} \sum_{j=0}^{1 / \eta\left[\left(\frac{r}{\sigma} / \sigma\right)^{\eta}\right] j}\right.$

$\left.-\mathrm{r} e^{-\left(\frac{r}{\sigma}\right)^{n}}\right]=0$

$\mathrm{R}\left(r_{i s}{ }^{*}\right)=\frac{A\left(\alpha Q_{i s}{ }^{*}+\beta\right)}{M+A\left(1-\gamma_{i}\right)\left(\alpha Q_{i s}{ }^{*}+\beta\right)}=e^{-\left(\frac{r}{\sigma}\right)^{\eta}}$

Hence,

If we put $\eta=1, \lambda=\frac{1}{\sigma}$, in equation (22), we found the lead time demand follows the Exponential distribution and it is found that the optimal values $Q_{i s}{ }^{*}$ and $r_{i s}{ }^{*}$ are given by:

$\alpha A Q_{i s}{ }^{* 2}-B-2 \mathrm{~A} \beta \quad\left\{r_{i s}{ }^{*}-\mu+\left(1-\gamma_{i}\right)\left[\frac{1}{\theta} e^{-\theta r}\right]\right\}-$ $2 \mathrm{M}\left[\frac{1}{\theta} e^{-\theta r}\right]=0$,

$\mathrm{R}\left(r_{i s}{ }^{*}\right)=\frac{A\left(\alpha Q_{i s}{ }^{*}+\beta\right)}{M+A\left(1-\gamma_{i}\right)\left(\alpha Q_{i s}{ }^{*}+\beta\right)}=e^{-\theta r}$

Which represent the optimal values when $\mathrm{X}$ follows the Exponential distribution with parameter $\theta$.

\section{Applications}

A businessman manages his import and export company in Egypt. He decided to import three electronic appliances (three products) from three different vendors. Table 8 in Appendix shows the values of the random variable of the lead time demand for 50 sample. The parameters are given in Table 1, Table 2 and
Table 3. Hence, a businessman wishes to get an optimal policy to minimize the expected total cost.

Table (1): The crisp parameters for multi-item

\begin{tabular}{cccc}
\hline & Item 1 & Item 2 & Item 3 \\
\hline $\bar{D}$ & 1000 & 900 & 810 \\
$C_{h i}$ & 10 & 25 & 35 \\
$C_{b i}$ & 20 & 30 & 40 \\
$C_{l i}$ & 30 & 35 & 45 \\
$p_{i}$ & 50 & 40 & 60 \\
$\theta_{i}$ & 0.5 & 0.4 & 0.8 \\
$\gamma_{i}$ & 0.7 & 0.67 & 0.56 \\
$K_{h i}$ & 1000 & 2000 & 2300 \\
\end{tabular}

Table (2): The fuzzy parameters for multi-item

\begin{tabular}{cccc}
\hline & Item 1 & Item 2 & Item 3 \\
\hline$\tilde{C}_{h i}$ & $(1,2,11,12)$ & $(1,2,26,27)$ & $(1,2,36,37)$ \\
$\tilde{C}_{b i}$ & $(1,3,21,23)$ & $(1,3,31,33)$ & $(1,3,41,43)$ \\
$\tilde{C}_{l i}$ & $(2,5,32,33)$ & $(2,5,37,38)$ & $(2,5,47,48)$ \\
$\widetilde{K}_{h i}$ & 780 & 1100 & 1200 \\
\hline
\end{tabular}

\section{Solution:}

By using One-Sample Kolmogorov-Smirnov Test, the data is fitted to Gamma distribution, where Table 4 shows the K-S statistic with their $\mathrm{P}$ value. If $\beta$ is a constant real number selected to provide the best fit of estimated expected total cost function and using the Mathematica program 9. Using the parameters of Tables 1, 2, 3 and 5in equations (4), (5), (9) and (10) to obtain the optimal solutions, $\lambda^{*}, Q^{*}$, $r^{*}$ and the minimum expected total cost for each item and source min $\left(\mathrm{E} T C_{i s}\right)$ is given by Table 7 at some different values of $\beta$, where the best fit of $\beta$ here is in $0<\beta \leq 1$ as shown in Table 6.

Table (4): one-sample Kolmogrov-Smirnov test of the lead time demand.

\begin{tabular}{lccc}
\hline & $\mathrm{X} 1$ & $\mathrm{X} 2$ & $\mathrm{X} 3$ \\
\hline Sample size & 50 & 50 & 50 \\
Statistic & 0.08098 & 0.09251 & 0.11868 \\
p-value & 0.872 & 0.75071 & 0.44764 \\
Level significance & 0.05 & 0.05 & 0.05 \\
$(\alpha)$ & & & \\
\hline
\end{tabular}

Table (5): The value of $\lambda^{*}$ for Item 1 and the first source at $\beta=0.1$

\begin{tabular}{ccc}
\hline $\boldsymbol{\lambda}$ & $\mathrm{E}(\mathrm{HC})$ & $\mathrm{E}\left(T C_{i}\right)$ \\
\hline 0 & 1237.08 & 10.9564 \\
0.5 & 1036.85 & 13.5533 \\
0.6304 & 1000.01 & 14.2241 \\
$\mathbf{0 . 6 3 0 5}$ & $\mathbf{9 9 9 . 9 8 1}$ & $\mathbf{1 4 . 2 2 4 7}$ \\
\hline
\end{tabular}


Table (6): The results of crisp and fuzzy values for Gamma distribution

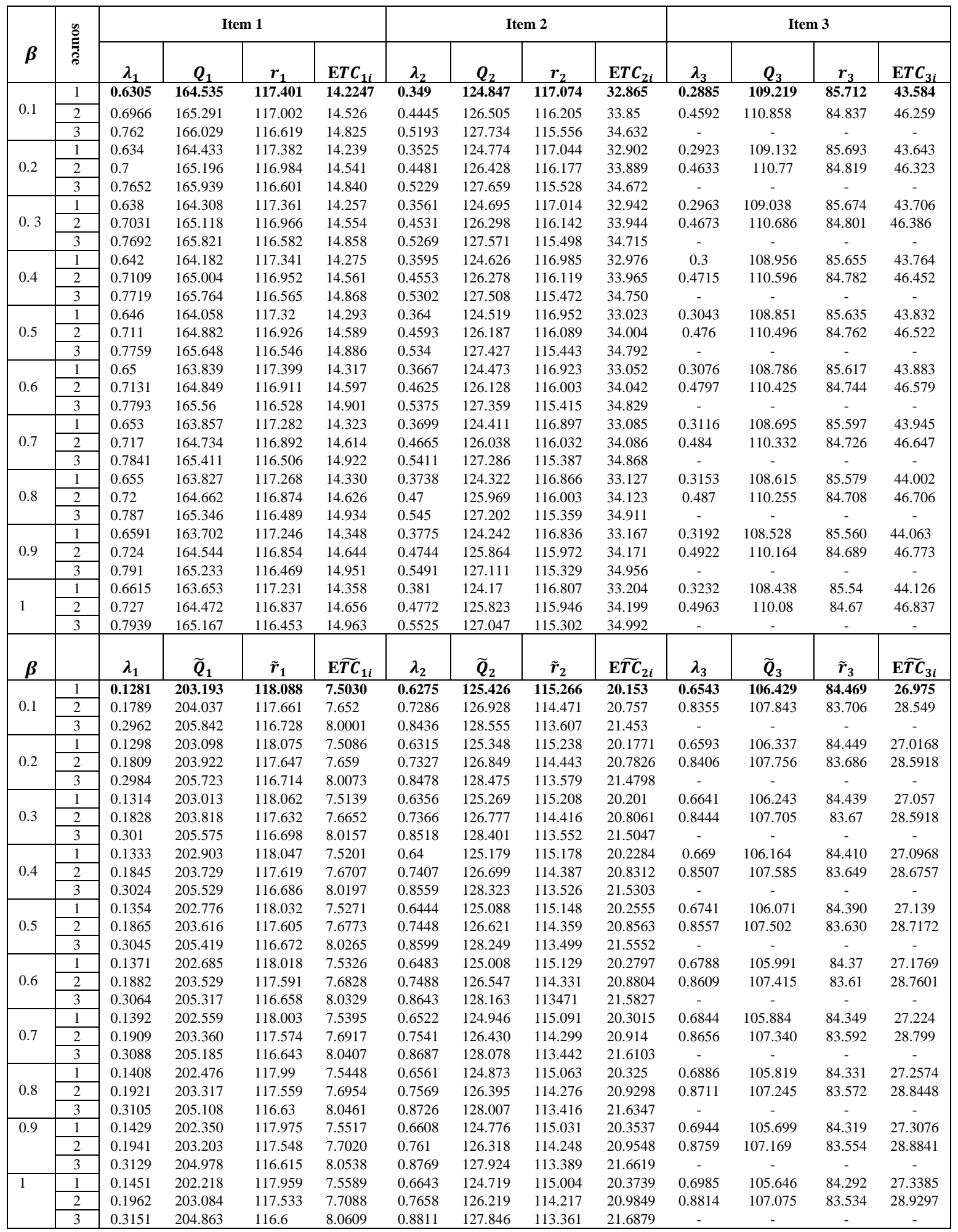

Table (7): The optimal policy of MIMS variables at $\beta=0.1$

\begin{tabular}{|c|cccc|c|c|c|c|c|c|}
\hline Item & $\lambda_{i s}{ }^{*}$ & $Q_{i s}{ }^{*}$ & $r_{i s}{ }^{*}$ & $E T C_{i s}$ & Source & $\lambda_{i s}{ }^{*}$ & $\tilde{Q}_{i s}{ }^{*}$ & $\tilde{r}_{i s}{ }^{*}$ & $\widetilde{E T C}_{i s}$ & Source \\
\hline 1 & 0.6305 & 164.535 & 117.401 & 14.2247 & 1 & 0.1281 & 203.193 & 118.088 & 7.5030 & 1 \\
\hline 2 & 0.349 & 124.847 & 117.074 & 32.865 & 1 & 0.6275 & 125.426 & 115.266 & 20.153 & 1 \\
\hline 3 & 0.2885 & 109.219 & 85.712 & 43.584 & 1 & 0.6543 & 106.429 & 84.469 & 26.975 & 1 \\
\hline Min TC & \multicolumn{10}{|c|}{90.6737} \\
\hline
\end{tabular}




\section{Conclusion and Future Work}

Upon studying the probabilistic multi item multi-source inventory model with varying holding cost under constraint using the Lagrange multipliers technique, the optimal order quantity $Q^{*}$ and the optimal reorder point $r^{*}$ for the $i^{\text {th }}$ item and $s^{\text {th }}$ sourceare introduced. Then, the minimum expected total cost $\min \mathrm{E}\left(T C_{i s}\right)$ for crisp and trapezoidal fuzzy number are deduced for each item and source. Also, we studied the model when the lead time demand follows Gamma, Weibull, Chi-square, Erlang and Exponential distributions. Also for an application we can determine the optimal source for each item. Finally, we found that the results in case fuzzy numbers are better than crisp numbers. In the future we will apply this model with constraints on the expected holding cost and the expected mixture shortage cost. Also, we will apply rough numbers for this model.

\section{References}

[1] Abuo-El-Ata M.O., Fergany H.A. andElwakeel M.F., (2003) "Probabilistic multi-item inventory model with varying order cost under two restrictions". International Journal of Production Economics, 83, 223-231.

[2] Azoury,K. and Brill, P.H. (1986): " An Application of The System-Point Method ToInventory Models Under Continuous Review". J. App. Prob.,23, pp.778-789.

[3] Bera, U. K., Rong, M., Mahapatra, N. K., and Maiti, M. (2009): "Amulti-item mixture inventory model involving random lead time and demand with budget constraint and surprise function". Applied Mathematical Modelling 33, 4337-4344.

[4] Chiang, J., Yao, J. S., and Lee, H. M. (2005): "Fuzzy inventory with backorder defuzzification by signed distance method". Journal of Information Science and Engineering, 21, 673-694.

[5] Fabrycky W.J. and Banks J. (1967) "Procurement and Inventory System: Theory and Analysis". Reinhold Publishing Corporation, USA.

[6] Fergany H.A. and Elwakeel M.F., (2004) "Probabilistic single-item inventory problem with varying order cost under two linear constraints".
Statistical Theory and MethodAbstracts,12(1),7181.

[7] Fergany H.A. and El-Saadani, M.E., (2005) "Constrained Probabilistic inventory model with continuous distributions and varying holding cost". International Journal of Applied Mathematics, Vol 17, No. 1, pp. 53-67.

[8] Fergany H.A., (2016):"Probabilistic multi-item inventory model with varying mixture shortage cost under restrictions". Springer plus, 5(1), 1-13.

[9] Fergany H.A., Hefnawy N.A. and Hollah O.M., (2014):" Probabilistic periodic review $\langle Q, r\rangle$ inventory model using lagrange technique and fuzzy adapting particle swarm optimization". Journal of Mathematics and Statistics.

[10] Kotb, K.A.M., Fergany, H.A., (2011):"Multiitem EOQ model with varying holding cost: a geometric programming approach". Int. Math. Forum 6.

[11] Gupta, P. K. and Hira, D. S. (1993): "Operations research" S. Chand \& Company Ltd New Delhi.

[12] Hadley, G. and Whitin, T.M., (1963) "Analysis of Inventory System". Prentice Hall, Inc., Englewood Cliffs, New Jersey.

[13] Hill, R. M. and Johansen, S. G. (2006): "Optimal and neer-optimal policies for lost sales inventory models with at most one replenishment order outstanding". European Journal of Operational Research, VoL 169, pp. 111-132.

[14] Padmanabhan, G. and Vrat, P. (1990): "Inventory model with a mixture of backorders and lost sales ". International Journal of Systems Sciences, Vol. 21: 1721-1726.

[15] Vijayan, T. and Kumaran M. (2008): "Inventory models with a mixture of backorders and lost sales under fuzzy cost". European Journal of operational Research, VoL 189, pp. 105-119.

[16] Yun, Z. and Xiaobo, Z. (2010): "A twodemand-class inventory system with lost sales and backorders". European Journal of Operational Research, Vol. 38, Issue.4:261-266. 